\title{
Contribution to optimize decision parameters in activated-sludge process using ANFIS model
}

\author{
Radia. Maachou ${ }^{1}$, Abdelouahab Lefkir ${ }^{2 *}$, Tarek Merabtene ${ }^{3}$, Amel Hamriche ${ }^{4}$, Abdemalek \\ Bermad $^{4}$ \\ ${ }^{1}$ USTHB, laboratory of faculty of chemistry, electrochemistry-corrosion, metallurgy and mineral \\ chemistry, BP 32 El-Allia, pc 16111, Algeria. \\ ${ }^{2}$ ENSTP, TPiTE Laboratory, Bp 32 cité sidi Garidi, Kouba, Algers, Algeria. \\ ${ }^{4}$ ENP, Civil engineering, Material and Environment Laboratory, Bp 32 Hassen-Badi 16000, Algeria.
}

\begin{abstract}
The monitoring of activated sludge processes is difficult because of their slow dynamics, the complexity of their behavior. To obtain the desired level of performance in an activated sludge system, a proper balance must be maintained between aeration (energy consumption) and the quantity of sludge recirculation. The objective of this study is to determine the necessary amount of recirculated sludge in activated sludge process in the aim to reduce the energy consumption with respect of environmental standards. To achieving this objective, a comparative study between three models was performed. In order to reduce the complexity of activated-sludge process, the first is a linear model based on principal component analysis method (PCA), the second is an Adaptive Neural Fuzzy Inference System model (ANFIS), while the third is based on coupling of those two approaches. The best values of validation criterion obtained with the last method show the high performance of the hybrid model proposed...
\end{abstract}

\section{Introduction}

Awareness of environmental problems caused by discharges from wastewater treatment plants and the will to improve and preserve the quality of receiving waters has led the adoption of higher regulatory standards, compliance with discharge standards, on the hand, and energy consumption consequent [1], on the other hand, are the key issue for managers of wastewater treatment plants.

In recent years, cost minimization has become increasingly important in the control and operation of wastewater treatment plants. In order to run plant economically efficient, operational costs such as pumping energy, aeration energy and dosage of different chemicals should be minimized. At the same time, the discharges to the recipient should be kept at a low level. Of course, minimizing the operational costs and at the same time treat the wastewater properly may lead to a conflict of interest that must somehow be solved [2].

The ASP is the most used in Algeria in the wastewater treatment process. Although the purification performance and reliability of this method are approved, several malfunctions

\footnotetext{
* Corresponding author: a lefkir06@yahoo.fr
} 
may occur due to the qualitative and quantitative changes of water to be treated, the aeration time in the aeration tanks, the amount of recirculated sludge [3].

In this context, the cost of the energy consumption during the aeration process is important. In general, approximately one third of the total operating cost of wastewater treatment plant (WWTP) is due to the energy requirements, and the energy consumed for the aeration represents approximately $60-65 \%$ of the total energy consumption $[4,5,6]$.

The aim of this work is focused on reduction of energy consumption on predicting the amount of recycle sludge necessary.

\section{Materials and methods}

\subsection{Activated sludge process}

The activated sludge process (ASP) is one of a number of unit processes used for the treatment of wastewaters. The basic feature of the process is the decomposition of complex dissolved and suspended organic substrates into simple end-products such as carbon dioxide and water. These systems involve inoculation with floc forming bacteria, which oxidize the organic matter, stabilizing the wastewater under aerobic conditions. A settling tank is used to separate the biomass constituents according to their settling abilities [7]. Decomposition is achieved by a heterogeneous culture of micro-organisms the activated sludge, which in part utilize the waste organic substrates in the synthesis of their own biological cell material $[8]$.

Many different processes happen simultaneously in wastewater treatment plants (WWTP), which were originally designed to reduce the biological oxygen demand, total suspended solids (SS) and nitrogen and phosphorus pollution [9]. The treatment plant generally includes a pre-treatment phase, during which the large elements (unrefined) are removed, followed by secondary treatment (or biological) to remove the organic material and dissolved. Finally, tertiary treatment is applied to destroy microorganisms.

After pre-treatment, the pre-treated water is directed to the aeration basins that are mixed with an aerated biomass and kept in suspension. The mixed liquor produced is composed of flocculated sludge and treated water directed to the clarifiers. The clarifier is an integral part of the activated sludge system. It has two main functions: it separates the biomass from the water in order to produce a good quality effluent free from settle able solids and it also thickens the biomass. In output thereof, the biomass is separated by decantation; part of the biomass is recycled (recycle sludge) to the basin because it contains numerous microorganisms which are needed for the reaction [10] to maintain an appropriate biomass concentration. The excess biomass is removed from the system and constitutes the secondary sludge that is drained and will be treated in a further sludge treatment process. Recycle sludge (RS) is a critical control variable as it redistributes the sludge between the secondary clarifier and the aeration tank, such that the healthy population of biomass is maintained in the aeration basin.

Thus, the operator must maintain a continuous return of activated sludge to the aeration tank or the process will show drastic decrease in performance. To obtain the desired level of performance in an activated sludge system, a proper balance must be maintained between aeration (energy consumption) and the quantity of sludge recirculation (pumped from the secondary clarifier back to the aeration tank), and the amount of excess sludge withdrawn from the system (usually pumped from the secondary clarifier towards sludge treatment). 


\subsection{Constraint of activated sludge process}

The dynamic behaviour of ASP is a result of many complex mechanisms [11] due to the specific growth rate of bacteria in biological process which varies with time and is influenced by many factors such as the substrate concentration, temperature, $\mathrm{pH}$, dissolved oxygen concentration, amount of inflow [12], imprecision and uncertainty of several parameters which are difficult to identify and strong nonlinearities. These changes may be the result of metabolic changes in biomass or of unpredictable genetic changes and unobservable from a macroscopic point of view [13]. Given all these factors, it is very difficult to construct a mathematical model for control of the process. In order to meet these demands, the use of advanced modelling methods by fuzzy logic model is required [14]. The basic components of Fuzzy Neuro model and techniques that have been used for their development will be discussed in the following.

\subsection{Fuzzy logic and neural networks}

Recently, soft computing method was proposed as a useful tool to model complex systems for process industries. In the last decades, the research on soft computing methods such as Neural network techniques and fuzzy logic fuzzy [15].

Artificial neural network technique is powerful because it can learn to represent complicated data pattern or the data relationship between input and output variables of the system being studied.

Fuzzy neural (FN) are hybrid systems that combine the theories of fuzzy logic and neural networks, thus can make effective use of easy interpretability of fuzzy logic, as well as superior learning ability and adaptive capability of neural networks.

A general fuzzy system has basically three components: fuzzification, fuzzy inference and defuzzification

1)The fuzzification consists in splitting in several terms the parameters which compose the characteristic vector. Each decomposition or term gets closer to a word of the natural language. For instance, the concentration can be "high", "medium" or "low". There exit two ways to set the fuzzification: a fuzzification with an equal distribution of the terms, and a fuzzification with a repartition adapted to the training data set.

The fuzzification of the input variables, which convert digital inputs available in fuzzy parts. It is then possible to associate variables of the membership the coefficients of the fuzzy subsets taking values in the range $[0,1]$.

2)The fuzzy inference is the process of formulating the mapping from given input(s) to output(s) using fuzzy logic. The mapping them provides a basis from which decisions can be made. The process of fuzzy inference involves all the topic such as fuzzification, defuzzification, implication and aggregation. It has found successful applications in a wide variety of fields, such as automatic control, data classification, decision analysis, expert systems, time series prediction, robotics, and pattern recognition. Because of its multidisciplinary nature, the fuzzy inference system is known by numerous others names, such as fuzzy-rule-based system, fuzzy expert system, fuzzy model, fuzzy associative memory, fuzzy logic controller and simply (and ambiguously) fuzzy system.

The basic structure of a fuzzy inference system consists of three conceptual components:

A rule base, which contains a selection of fuzzy rules;

A database (or dictionary), which defines the membership functions used in the fuzzy rules;

And a reasoning mechanism, which performs the inference procedure upon the rules and given facts to derive a reasonable output or conclusion. 
We conclude that fuzzy inference is consisted by the basic rules and the database. The combination of inputs with the fuzzy rules allows drawing conclusions. (Produced the image of fuzzy part stemming the fuzzification, by a fuzzy relation usually built from rules.

For example:

If $\left(\left(\mathrm{Y}_{\mathrm{BOD}}\right.\right.$ is high) and $\left(\mathrm{Y}_{\mathrm{COD}}\right.$ is high) and $\left(\mathrm{Y}_{\mathrm{SS}}\right.$ is high) and $\left(\mathrm{Y}_{\mathrm{SS}}\right.$ is high)) then (Ren is high)

If $\left(\left(\mathrm{Y}_{\mathrm{BOD}}\right.\right.$ is low) and ( $\mathrm{Y}_{\mathrm{COD}}$ is low) and ( $\mathrm{Y}_{\mathrm{SS}}$ is low) and ( $\mathrm{Y}_{\mathrm{SS}}$ is low)) then (Ren is low) if ((RS is high) and ( $Y_{C O D}$ is high) and $\left(Y_{S S}\right.$ is high)) then (Ren is high)

3)Defuzzification: The inference methods provide a resulting membership function for the output variable, so it is a fuzzy information. By this step, is then made the return in the real sizes of output. It is for this purpose calculated from the degrees of membership in all fuzzy sets of the output variable, the abscissa corresponding to the value of this output, so it converts the blurs on outputs inference mechanism in digital outputs. In other words, where the linguistic fuzzy output has to be translated into a numerical value such as the necessary recycle sludge.

ANFISs were first proposed by Jang in 1992. These are adaptive networks whose function is similar to FISs. The aim of an ANFIS is to integrate the best features of fuzzy systems and NNs. The advantages of an ANFIS over an FIS are as follows:

-An ANFIS can simulate and analyze the mapping relation between input and output data through a learning algorithm to optimize the parameters of a given FIS.

-An FIS is fully dependent on its membership functions. An ANFIS involves networks which combine nodes and directional links, and some learning rules are associated with these networks. The networks learn relationships between inputs and outputs.

-An ANFIS can be trained without any need for the expert knowledge usually required for the standard fuzzy logic design [16] (Tyagi K et al, 2014).

\section{Results and discussions}

Database collection of the downstream and upstream from the station was conducted. The series of data was collected daily from January 2006 until December 2013. In addition, 250 daily data yields describing the pollution control were collected during a weekly measurement.

ANFIS model is applied to reduce the energy consumption. The data of Recycle sludge were separated into two subsamples: the training data and validation data. The training data were chosen among filtered data where elimination of NO3- observed, and the validation data were chosen among unfiltered data of NO3 where excess of NO3 observed. The input parameters such as the removal yield of Suspended Solids (RSS), Biological oxygen demand (YBOD), Chemical oxygen Demand (YCOD) were selected to represent the pollution and RS was selected to present the amount of recycle sludge, this last is divided on the maximum value. The resulting values are normalized (between 0 and 1). Decision parameters is energy ratio.

\subsection{The membership functions}

Development of the fuzzy neural network model involves using both fuzzy logic and neural networks. Here, the fuzzy logic is really a matter of the fuzzy set theory particularly used to deal with process imprecise infonnation with a changed membership function. This fuzzy logic technique uses non-conventional membership function that is different than the classical membership function that takes only two values: one, when an element belongs to the set, and zero, when it does not. Because fuzzy sets describe vague concepts based on the premise that the elements used are not numbers but belong to words or the value of a linguistic variable, an element of a fuzzy set naturally belongs to the set with membership values from 
the interval [0,1]. A fuzzy membership function associated with a given fuzzy set maps an input value to its appropriate membership value [17].
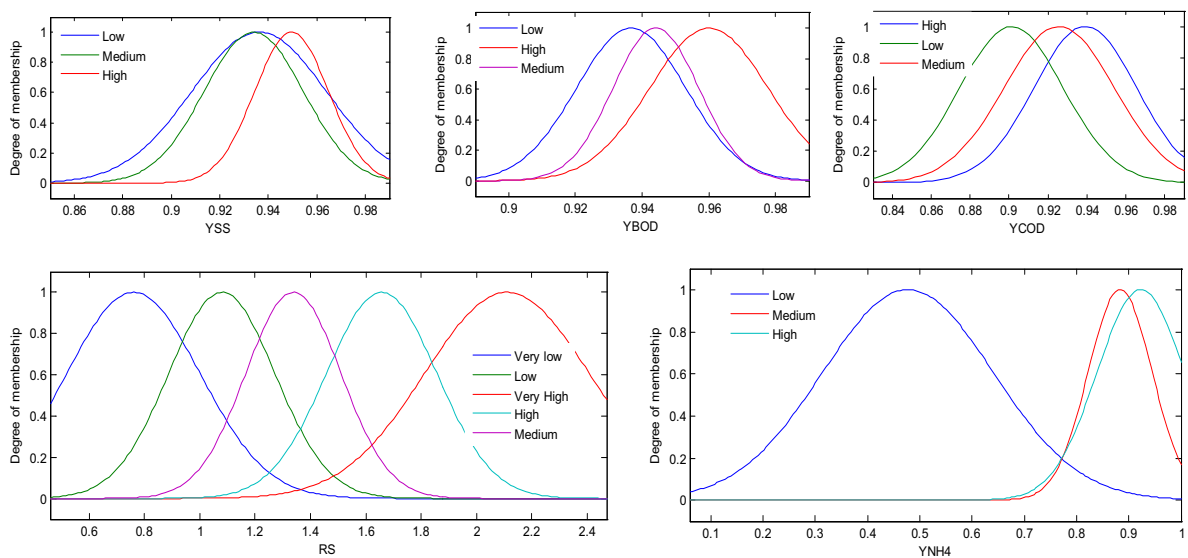

Fig 1. Example of membership functions for the input and output variables.

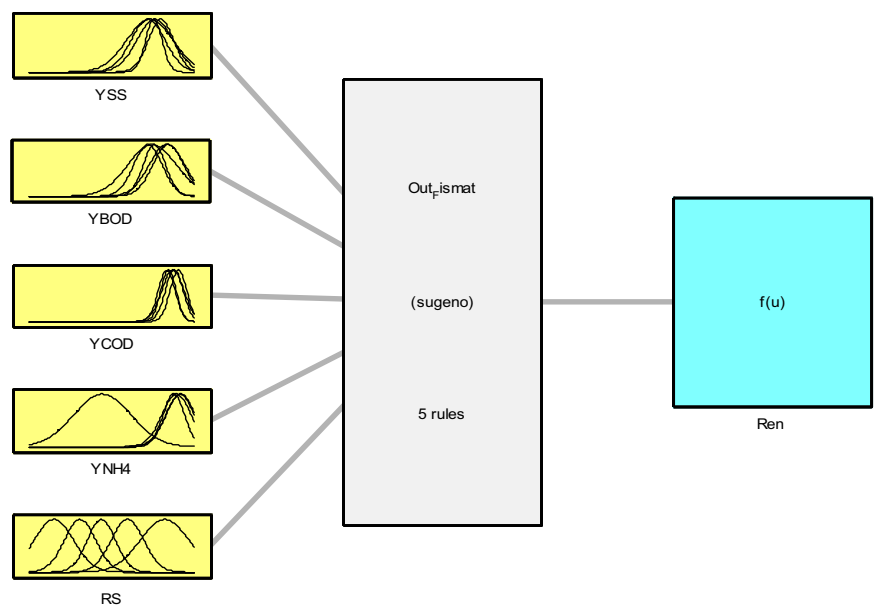

System Outismat: 5 inputs, 1 outputs, 5 rules

Fig 2. Sugeno fuzzy inference system (FIS) system.

\subsection{Simulation results}

In the first part, ANFIS model has been proposed and applied to simulate recycle sludge (RS) in activated sludge process in objective to optimize the energy consumption given by the fig3.

We symbolize Ratio energy by Ren/ Ren [kWh / m3] = energy consumption [kWh / day] / input flow [m3 / day].

In order to evaluate the predicting performance of ANFIS, the Root Mean Square Error criterion (RMSE) is used to calculate the difference between the simulated and observed values and the correlation coefficient $\mathrm{R}[14]$ are given in table 1: 
The correlation coefficient allows assessment of the degree of relationship while the RMSE criterion used to measure the distortion between the observed and the simulated values. We concluded that the coupling of the two criteria is necessary for a proper assessment model.

Simulation and prediction have shown that the resulting model can adequately predict the process with satisfactory results. These last were obtained during the learning and validation periods, revealing the advantages of fuzzy reasoning and justifying the predictive power of the model developed to simulate the amount of the recycle sludge in activated sludge process [14].

Table 1. Validation criteria of learning and validation periods.

\begin{tabular}{|l|l|l|}
\hline & validation criteria & Values \\
\hline \multirow{2}{*}{ Learning period } & $\mathrm{RMSE}$ & 11,2 \\
\cline { 2 - 3 } & $\mathrm{R}(\%)$ & 83,37 \\
\hline
\end{tabular}

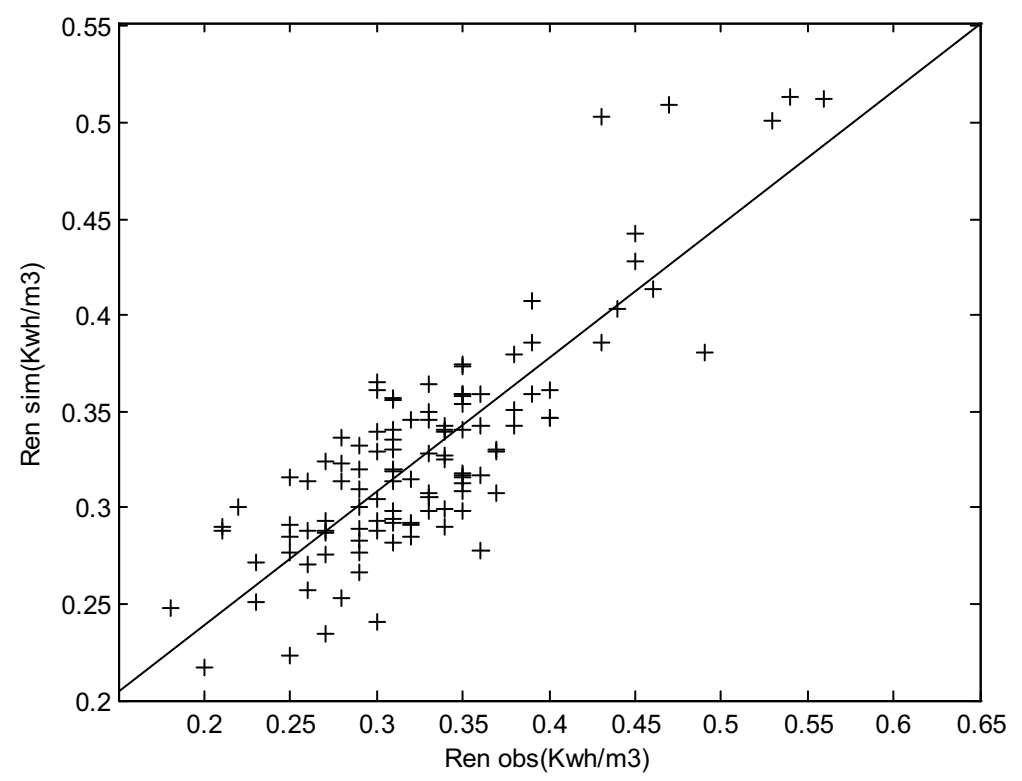

Fig 3. Correlation line between Ren (Obs) and Ren (Sim)

In the second part, the reduction of energy was given by the fig 2

The variation of the observed and simulated energy ratio based on the learning sample is given by a histogram shown in fig 4 .

According to the histogram plot, we find significant differences between the observed values and simulated values of Ren, this confirms that there is energy consumption.

The optimized Ren percentage equal to $14.34 \%$ 


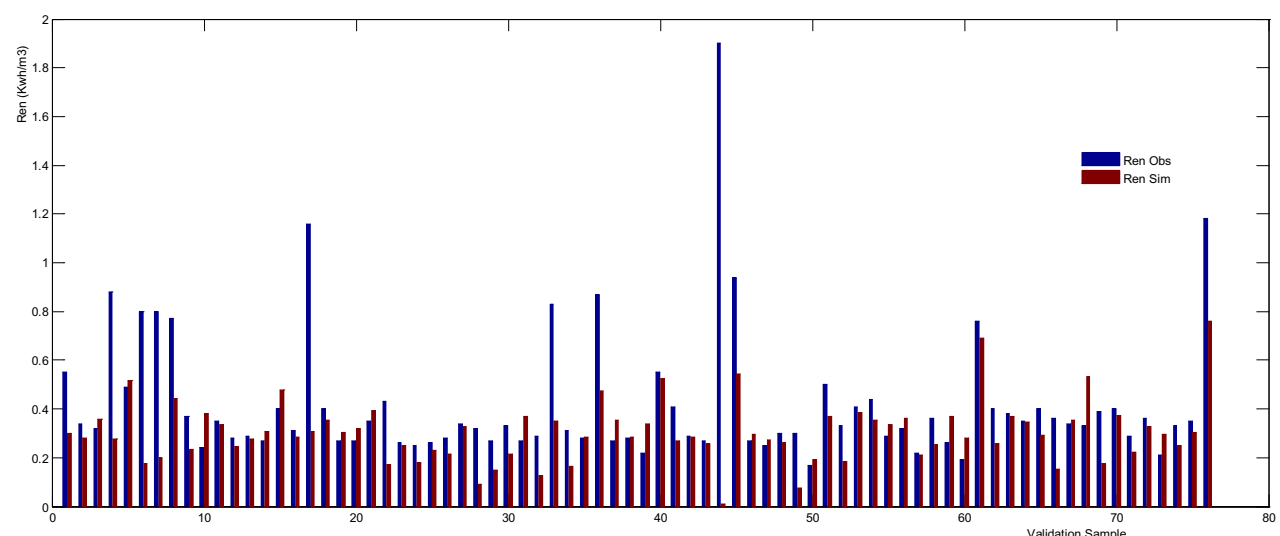

Fig 4. Comparison between energy ratio observed \& optimized for sample validation.

\section{Conclusions}

The present study concludes that the application of ANFIS can improve Sludge recycling system, providing the optimal yields. ANFIS indicates the high ability of modelling a more complicated process such as the activated sludge processes, for improving the performance of wastewater treatment plant and can lead to a better understanding of the system. However, the complexity and uncertainty in the process make the task somewhat complicated using traditional deterministic models.

If the economy is only a few percent, it can be very significant in terms of $\mathrm{kWh}$ per year per decade.

\section{References}

1. Holmberg A., Modelling of the activated sludge process for microprocessor-based state estimation and control, water research 16, 1233-1246, (1982).

2. P. Samuelsson, B. Halvarsson, Bengt Carlsson, cost efficient operation of a denitrifying activated sludge process, Water Research., 41, P2325 - 2332, (2007)

3. Fernandez FJ., Castro MC., Rodrigo M.A., Canizares P., Reduction of aeration costs by tuning a multi-set point on/off controller: A case study, Control Engineering Practice 19, 1231-1237 (2011).

4. Rieger L., Alex J., Gujer W., \& Siegrist H., Modelling of aeration systems at wastewater treatment plants. Water Science \& Technology, 53, 439-447 (2006)

5. Duchène Ph., Cotteux E., \& Capela S., Applying fine bubble aeration to small aeration tanks. Water Science \& Technology, 44(2-3), 203-210 (2001).

6. Rustom R, Modelling Activated Sludge Wastewater Treatment Plants Using Artificial Intelligence Techniques (Fuzzy Logic and Neural Networks), thèse doctorat, Heriot Watt University, UK (2009).

7. Mafalda C., Sarraguça et al., Quantitative monitoring of an activated sludge reactor using on-line UV-visible and near-infrared spectroscopy, Anal Bioanal Chem.,395,11591166, (2009).

8. Tong R. M, Beck M. B., Latten A.,, fuzzy control of the activated sludge wastewater treatment process, aulomatica, vol. 16, pp. 659701 pergamon press, (1989)

9. Lefkir A., Maachou R., Bermad A., Khouider A., Factorization of physicochemical parameters of activated sludge process using the principal component analysis, Desalination and Water Treatment, 1-6 (2015). 
10. Chen $\mathrm{Y}$, Zhiqiang $\mathrm{C}$, Abraham K, application of fuzzy reasoning to the control of an activated sludge plant*, fuzzy sets and systems 38 1-14 (1990)

11. Ruano M.V., J. Ribes ., Sin G., Seco A., Ferrer J., A systematic approach for fine-tuning of fuzzy controllers applied to WWTPs, Environmental Modelling \& Software 25, 670$676(2010)$

12. Bououden S., Chadli M.,.Karimi H.R. 2015, Control of uncertain highly nonlinear biological process based on Takagi-Sugeno fuzzy models, Signal Processing, 108-205 Mafalda et al., (2009).

13. Grizale P V H, Modélisation et commande floues de type Takagi-Sugeno appliquées à un bioprocédé de traitement des eaux usées, thèse d'état, Automatic. Université Paul Sabatier, Toulouse III, (2007)

14. Maachou R., Lefkir A., Khouider A. And Bermad A., control of recycle sludge in activated sludge process using adaptive neuro-fuzzy logic controller (anfis) proceedings of the 14th international conference on environmental science and technology rhodes, greece, 3-5 september, (2015)

15. Qiao J, Li W, Han H, Soft Computing of Biochemical Oxygen Demand Using an Improved T-S Fuzzy Neural Network Chinese Journal of Chemical Engineering 22 (2014) 1254-1259, (2014).

16. Tyagi K, Arun Sharma., An adaptive neuro fuzzy model for estimating the reliability of component-based software systems , 38-51, Applied Computing and informatics, (2014) 